\title{
Traumatic brain injury increases the risk of psychiatric illness
}

Fann JR, Burington B, Leonetti A, et al. Psychiatric illness following traumatic brain injury in an adult health maintenance organization population. Arch Gen Psych 2004;61:53-61.

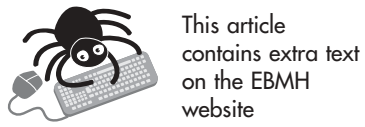

Does traumatic brain injury increase the risk of psychiatric illness?

\section{METHODS}

$\square$

Design: Prospective cohort study

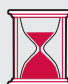

Follow up period: Three years.

Setting: Puget Sound area, Washington State, USA; recruited in 1992.

으 People: 939 people, aged $>15$ years, diagnosed with traumatic brain injury according to ICD-9-CM criteria. For each case of traumatic brain injury three people matched for sex, age, and diagnosis date were selected as controls. Exclusions: incomplete medical records for the preceding year; previous traumatic brain injury.

1

Risk factors: Mild traumatic brain injury; moderate to severe traumatic brain injury (ICD-9-CM)

Outcomes: Incidence of any psychiatric disorder determined by diagnosis (ICD-9-CM), need for psychiatric medication and use of psychiatric services.

\section{MAIN RESULTS}

After one year, prevalence of psychiatric illness significantly increased in people with moderate to severe and mild traumatic brain injury, compared with controls (moderate to severe: $49 \%$; mild: 34\%; control: 18\%; see table 1, http://www.ebmentalhealth.com/ supplemental). People with prior psychiatric illness are more likely to suffer from a psychiatric illness after traumatic brain injury. The effect of TBI on subsequent psychiatric illness, however, is greater among those without prior psychiatric illness (see table 2, http:/ www.ebmentalhealth.com/supplemental).

\section{CONCLUSIONS}

People with both moderate to severe and mild traumatic brain injury are at increased risk of psychiatric illness.

For correspondence: Jesse $R$ Fann, Department of Psychiatry and Behavioral Sciences, University of Washington, Seattle, WA, USA; fann@ u.washington.edu

Sources of funding: the National Center for Injury Prevention and Control, Centers for Disease Control and Prevention, Atlanta, GA, USA.

\section{Commentary}

The study by Fann et al looking at psychiatric illness following traumatic brain injury (TBI) is important for a number of reasons.

Firstly, TBI accounts for most cases of permanent disability after trauma, given that trauma injuries occur most frequently in the young $(<45$ years) the cost, both to the individual and to society is enormous. Secondly, the psychological sequelae of TBI may frequently be overlooked and as such, under treated. Nowhere is this more apparent than in people with mild $\mathrm{TBI}$, who comprise over $85 \%$ of all traumatic brain injuries, and who routinely do not receive adequate follow up care. ${ }^{2}$

The present study benefits from a large sample size, prospective cohort design, and a clear distinction between people with mild TBI and those with moderate or severe injuries, the latter point being frequently ignored in earlier research. These three factors, together with careful attention to putative antecedent risk factors for poor outcome, allow the authors to address one of the enduring conundrums in neuropsychiatry: why do so many people with a TBI deemed mild have persistent, disabling symptoms? One answer, as these data make clear, is the greater potential for people with mild as opposed to moderate or severe brain injuries to develop affective disorders. In addition, people with premorbid psychiatric histories appear to be particularly vulnerable. These results dovetail with those from other researchers who have shown that the disabling affects of mood disorders in the general population are present and possibly amplified in people with mild TBI. ${ }^{3}$ Given the association between TBI, mood disorders, and cognitive dysfunction, these findings take on added salience. ${ }^{4}$

These new insights into the deleterious effects of TBI suggest a fresh approach to treatment. To date, studies that have attempted routine multidisciplinary treatment of all people with TBI have delivered poor results, most notably in the mild category. ${ }^{56}$ The large number of people with mild TBI also makes such an approach unpractical. However, targeting those patients with a premorbid mental health history coupled with early post injury psychiatric intervention may deliver a more promising therapeutic yield.

Anthony Feinstein, MPhil, PhD, FRCPC Department of Psychiatry, University of Toronto and Sunnybrook and Women's College Health Sciences Center, Toronto, Canada

1 Jennett B. Epidemiology of head injury. J Neurol Neurosurg Psychiatry 1996;60:362-9.

2 Feinstein A, Rapoport M. Mild traumatic brain injury: the silent epidemic. Can J Pub Health 2000:91:325-6.

3 Rapoport M, McCullagh S, Streiner D, et al. The clinical significance of mood disorders following mild traumatic brain injury. Psychosomatics 2003;44:31-7

4 Fann JR, Uomoto JM, Katon WJ. Cognitive improvement with treatment of depression following mild traumatic brain injury. Psychosomatics 2001;42:48-54.

5 Paniak C, Toller-Lobe G, Durand A, et al. A randomized trial of two treatments for mild traumatic brain injury. Brain Injury 1998;12:1011-23.

6 Wade DT, Crawford S, Wendon FJ, et al. Does routine follow-up after head injury help? A randomised controlled trial. J Neurol Neurosurg Psychiatry 1997;62:478-84. 